September 2012

Revised: June 2013

\title{
Hidden sector dark matter explains the DAMA, CoGeNT, CRESST-II and CDMS/Si experiments
}

\author{
R. Foot ${ }^{1}$ \\ ARC Centre of Excellence for Particle Physics at the Terascale, \\ School of Physics, University of Melbourne, \\ Victoria 3010 Australia
}

\begin{abstract}
We examine data from the DAMA, CoGeNT, CRESST-II and CDMS/Si direct detection experiments in the context of multi-component hidden sector dark matter. The models considered feature a hidden sector with two or more stable particles charged under an unbroken $U(1)^{\prime}$ gauge interaction. The new gauge field can interact with the standard $U(1)_{Y}$ via renormalizable kinetic mixing, leading to Rutherford-type elastic scattering of the dark matter particles off ordinary nuclei. We consider the simplest generic model of this type, with a hidden sector composed of two stable particles, $F_{1}$ and $F_{2}$. We find that this simple model can simultaneously explain the DAMA, CoGeNT, CRESST-II and CDMS/Si data. This explanation has some tension with the most recent results from the XENON100 experiment.
\end{abstract}

\footnotetext{
${ }^{1}$ E-mail address: rfoot@unimelb.edu.au
} 


\section{Introduction}

The existence of non-baryonic dark matter, is clearly indicated by observations on small and large astronomical scales. In particular, the observed flat rotation curves in spiral galaxies, CMB anisotropies, and matter power spectrum suggest the existence of a cold (or warm) dark matter component with energy density around five times larger than ordinary baryons[1]. These inferences aside, the precise nature of dark matter is not yet known. If dark matter interacts with ordinary matter then it might be directly detectable with Earth based experiments, some of which have yielded some impressive results. Most notable, are the DAMA/NaI [2] and DAMA/LIBRA [3] experiments. These experiments have observed an annual modulation in the 'single hit' event rate, over more than 12 annual cycles, with phase consistent with expectations from dark matter interactions[4]. Over the last few years, the CoGeNT experiment $[5,6]$, using a p-type point-contact Germanium detector with very low energy threshold, has observed a rising event rate at low energies. These events could not be explained by known backgrounds and provide evidence supporting the DAMA signal. Most recently, the CRESST-II[7] and CDMS/Si[8] experiments have obtained results that are also compatible with a dark matter signal rising at low energies.

A satisfactory explanation of these experiments requires a specific model for dark matter. One promising approach appears to be in the framework of multi-component hidden sector models. In this scenario dark matter arises from a multi-component hidden sector which contains an unbroken $U(1)^{\prime}$ gauge interaction. The new gauge field is presumed to interact with the standard $U(1)_{Y}$ via renormalizable kinetic mixing, leading to Rutherford-type elastic scattering of the dark matter particles off ordinary nuclei. The specific case where the hidden sector is isomorphic to the ordinary sector, mirror dark matter $[9,10]$, has also been discussed at length, and found to be compatible with the DAMA, CoGeNT, CRESST-II and CDMS/Si results[11, 12, 13, 14, 15]. More generally, though, these works indicate that there is a larger class of such hidden sector models which are capable of explaining the experiments. In this article we examine in detail the simplest such multi-component hidden sector model. It serves as a useful prototype of generic dark matter models of this kind.

The outline of this article is as follows. Section 2 reviews some general aspects of the chosen hidden sector framework. Section 3 examines the specific case of the model with two stable hidden sector particles, $F_{1}$ and $F_{2}$. Section 4 confronts this two component hidden sector model with the latest data from DAMA, CoGeNT, CRESST-II and CDMS/Si experiments. Section 5, follows up on an example near the combined best fit identified in section 4 . The compatibility of this example with XENON100 data is looked at in section 6. Finally, in section 7 we conclude. 


\section{Hidden sector dark matter}

The models considered relegate dark matter particles to a generic hidden sector with an unbroken $U(1)^{\prime}$ gauge interaction. The special case where the hidden sector is isomorphic to the standard model sector - mirror dark matter - has been discussed in the context of direct detection experiments in ref.[11, 12, 13, 15]. Here we examine the more generic hidden sector case. This includes models with broken mirror symmetry[16] and potentially many other hidden sector models. Somewhat related models have also been studied in the recent literature e.g.[17]. More generally, hidden sector models with light stable particles can also be motivated from the similar inferred energy density of the ordinary and dark matter in the Universe, see e.g.[18]. Although cosmological and astrophysical discussions are beyond the scope of this paper, we refer the reader to recent work[19] which indicates that dissipative hidden sector dark matter can be rich enough to explain the puzzling regularities observed in spiral galaxies (cored dark matter density profile etc)[19]. Also, such models might potentially be consistent with observations of elliptical galaxies[19], the bullet cluster[20,19] and other astrophysical observations, e.g.[21].

We consider spin-independent elastic scattering of dark matter particles on ordinary nuclei. The dark matter particles are assumed to arise from a hidden sector which contains an unbroken $U(1)^{\prime}$ gauge interaction and possibly other gauge interactions. This means that the interactions of the theory are described by the Lagrangian:

$$
\mathcal{L}=\mathcal{L}_{S M}\left(e, \nu, u, d, B_{\mu}, \ldots\right)+\mathcal{L}_{\text {dark }}\left(F_{1}, F_{2}, . ., F_{N}, B_{\mu}^{\prime}, \ldots\right)+\mathcal{L}_{\text {mix }}
$$

where $\mathcal{L}_{\text {mix }}$ contains possible interactions connecting ordinary and hidden sector particles. An example of $\mathcal{L}_{\text {dark }}$, with $N$ Dirac fermions $F_{j}, j=1, \ldots, N$, is

$$
\mathcal{L}_{\text {dark }}=-\frac{1}{4} F_{\mu \nu}^{\prime} F^{\prime \mu \nu}+\bar{F}_{j}\left(i D_{\mu} \gamma^{\mu}-m_{j}\right) F_{j}
$$

where $D_{\mu}=\partial_{\mu}+i g^{\prime} B_{\mu}^{\prime} Q^{\prime}$ is the covariant derivative and $j$ is summed over $1, \ldots, N$. In this example the $N$ particles $F_{j}$ are each absolutely stable due to the presence of $U(1)_{1} \otimes U(1)_{2} \otimes \ldots . \otimes U(1)_{N}$ global symmetries. Importantly these symmetries are not imposed; they are accidental, like the $U(1)_{B}$ and $U(1)_{L}$ global symmetries of the standard model. Evidently, having dark matter arise from a hidden sector is theoretically attractive because it is a natural way to introduce dark matter particles which are dark, massive, stable and importantly do not modify standard model physics.

Dark matter elastic scattering depends on the cross-section, $d \sigma / d E_{R}$, and on the halo distribution function, $f_{F_{j}}$, of the dark matter species (taking the general case of $N$ stable species). Both of these quantities depend crucially on the particle physics. Considering first the cross-section, this can have different recoil energy dependence depending on the type of interaction. The most common form discussed in the literature is contact interactions which give an energy independent cross-section (excepting here the energy dependence due to the form factor). However, we consider Rutherford scattering which features non-trivial energy dependence, $d \sigma / d E_{R} \propto 1 / E_{R}^{2}[12]$. From the point of view of hidden sector dark matter, where the hidden sector contains an 
unbroken $U(1)^{\prime}$ gauge interaction, the latter appears to be the most natural form of interaction since it arises from $U(1)^{\prime}-U(1)_{Y}$ kinetic mixing which is both gauge invariant and renormalizable. Rutherford scattering results because the interaction is mediated by a massless particle (the photon). ${ }^{2}$

The distribution function, $f_{F_{j}}$, also depends on the particle physics. For dark matter composed of only one type of particle with mass, $m$, the distribution is typically assumed to be Maxwellian, with an effective temperature set by the galactic rotational velocity: $T \approx \frac{1}{2} m v_{r o t}^{2}$. This leads to a distribution: $f=\exp \left(-v^{2} / v_{0}^{2}\right)$ with $v_{0} \approx v_{\text {rot }}$. On the other hand, if dark matter is multi-component and self-interacting both plausible if dark matter arises from a hidden sector with unbroken $U(1)^{\prime}$ gauge interaction, then the distribution function is still Maxwellian, but the velocity dispersion depends on the mass of the species. $v_{0}\left[F_{j}\right]=\sqrt{2 T / m_{j}}$ (recall the index $j$ labels the stable particle species, $j=1, \ldots, N)$. If the interactions are frequent enough so that the particles in the halo have a common (local) temperature ${ }^{3}, T$, then it follows that the velocity dispersion depends on $m_{j}$ via $v_{0}\left[F_{j}\right] \propto 1 / \sqrt{m_{j}}$. In fact assuming an isothermal pressure supported halo in hydrostatic equilibrium, one can show that[13]

$$
v_{0}\left[F_{j}\right]=v_{r o t} \sqrt{\frac{\bar{m}}{m_{j}}}
$$

where $\bar{m}=\sum n_{j} m_{j} / \sum n_{j}$ is the mean mass of the particles in the halo. Thus the velocity dispersion can vary greatly depending on the masses within the model.

\section{Two component hidden sector dark matter}

Consider the case where the hidden sector contains two stable $U(1)^{\prime}$ charged dark matter particles $F_{1}$ and $F_{2}$ with masses $m_{F_{1}}$ and $m_{F_{2}}\left[F_{1}\right.$ and $F_{2}$ can be fermionic as in the example Eq.(2) or alternatively bosonic]. If their abundance in the Universe arises from a particle-antiparticle asymmetry then two stable particles with opposite in sign, but not necessarily equal in magnitude, $Q^{\prime}$ charges is actually the minimal case given $Q^{\prime}$ neutrality of the Universe. In fact this neutrality is not just global, $U(1)^{\prime}$ electric fields will ensure local neutrality of a plasma containing $F_{1}$ and $F_{2}$ particles. That is,

$$
n_{F_{1}} Q_{F_{1}}^{\prime}+n_{F_{2}} Q_{F_{2}}^{\prime}=0
$$

where $n_{F_{j}}\left[Q_{F_{j}}^{\prime}\right]$ is the local number density $\left[U(1)^{\prime}\right.$ charge] of $F_{j}$.

\footnotetext{
${ }^{2}$ The case where the mediator is light is also possible, and has been studied recently in ref.[22]. As shown there, in the context of the DAMA, CoGeNT and CRESST-II experiments, Rutherford scattering arises if the mediator has mass much less than $10 \mathrm{MeV}$, while point-like interaction arises if the mediator has mass much greater than $10 \mathrm{MeV}$.

${ }^{3}$ This assumes that the time scale for self interactions in the Milky Way galaxy is much shorter than cosmological time scales. This assumption is generally valid if the interactions have similar strength to those of the ordinary charged particles, see e.g.[21].
} 
We assume that the $U(1)^{\prime}$ gauge field interacts with the standard $U(1)_{Y}$ gauge field via the gauge invariant and renormalizable kinetic mixing interaction[23]:

$$
\mathcal{L}_{\text {mix }}=\frac{\epsilon^{\prime}}{2 \cos \theta_{w}} F^{\mu \nu} F_{\mu \nu}^{\prime}
$$

where $F_{\mu \nu}$ is the standard $U(1)_{Y}$ gauge boson field strength tensor, and $F_{\mu \nu}^{\prime}$ is the field strength tensor for the hidden sector $U(1)^{\prime}$. This interaction enables the hidden sector $U(1)^{\prime}$ charged particles $F_{j}$ to couple to ordinary photons[24] with electric charge $g^{\prime} Q_{F_{j}}^{\prime} \epsilon^{\prime} \equiv \epsilon_{F_{j}} e$. [Henceforth we define $\epsilon \equiv \epsilon_{F_{2}}$ ]. The cross-section of a $F_{2}$ particle to elastically scatter off an ordinary nucleus, $A$, presumed at rest with atomic number $Z$, is given by[12]: ${ }^{4}$

$$
\frac{d \sigma}{d E_{R}}=\frac{\lambda}{E_{R}^{2} v^{2}}
$$

where

$$
\lambda \equiv \frac{2 \pi \epsilon^{2} Z^{2} \alpha^{2}}{m_{A}} F_{A}^{2}\left(q r_{A}\right)
$$

and $F_{A}\left(q r_{A}\right)$ is the form factor which takes into account the finite size of the nuclei. Helm proposed a simple analytic expression for the form factor, which we adopt in our numerical work $[25,26]$.

Rotation curve data in spiral galaxies suggests that the $F_{1}$ and $F_{2}$ particles are (roughly) spherically distributed in a pressure supported halo [c.f. ref.[27]]. We assume that the self-interactions are frequent enough as to thermalize the distributions of $F_{1}$ and $F_{2}$ with a common temperature $T$. We further assume that the binding energy of atomic bound states containing $F_{1}$ and $F_{2}$ particles are much less than this temperature, so that the $F_{1}$ and $F_{2}$ particles can be treated as two components of a plasma ${ }^{5}$. Typically this requires $\alpha^{\prime 2} m_{F_{1}} m_{F_{2}} /\left(m_{F_{1}}+m_{F_{2}}\right) \ll \mathrm{keV}$ where $\alpha^{\prime} \equiv g^{\prime 2} Q_{F_{1}}^{\prime} Q_{F_{2}}^{\prime} / 4 \pi$.

As mentioned earlier, the condition of hydrostatic equilibrium relates the temperature of the particles to the galactic rotational velocity, $v_{\text {rot }}$, resulting in a mass dependent velocity dispersion, Eq.(3). In the two component case the mean mass of the particles in the galactic halo is given by

$$
\begin{aligned}
\bar{m} & \equiv \frac{n_{F_{1}} m_{F_{1}}+n_{F_{2}} m_{F_{2}}}{n_{F_{1}}+n_{F_{2}}} \\
& =\frac{m_{F_{1}}-\frac{Q_{F_{1}}^{\prime}}{Q_{F_{2}}} m_{F_{2}}}{1-\frac{Q_{F_{1}}^{\prime}}{Q_{F_{2}}^{\prime}}}
\end{aligned}
$$

\footnotetext{
${ }^{4}$ Unless otherwise indicated, natural units where $\hbar=c=1$ are assumed.

${ }^{5}$ The alternative case, where $F_{1}$ and $F_{2}$ are tightly bound into atoms has been discussed recently in ref.[28]. In the tightly bound limit, the interaction between ordinary matter and such atomic dark matter becomes point-like, and its implications for direct detection experiments are quite different from the case we study here.
} 
where we have made use of Eq.(4). From the point of view of direct detection experiments the interesting region of parameter space is where $m_{F_{1}} \ll m_{F_{2}}$ and $\left|Q_{F_{1}}^{\prime}\right| \ll\left|Q_{F_{2}}^{\prime}\right|$ so that $\bar{m} \ll m_{F_{2}}$. It then follows from Eq.(3) that $v_{0}^{2}\left(F_{2}\right) \ll v_{r o t}^{2}$. The narrow velocity dispersion (recall $\sigma_{v}^{2}=3 v_{0}^{2} / 2$ ) can greatly reduce the rate of $F_{2}$ interactions in higher threshold experiments such as XENON100[29] and CDMS/Ge [30] whilst still allowing $F_{2}$ to explain the signals in the lower threshold DAMA and CoGeNT experiments. The $F_{1}$ state can be too light to be directly detected in the experiments (roughly this means that $m_{F_{1}} \lesssim 5 \mathrm{GeV}$ ), but influences the way $F_{2}$ interacts due to its effect on the velocity dispersion of $F_{2}$. With these assumptions, current experiments depend on three parameters, $m_{F_{2}}, \bar{m}, \epsilon \sqrt{\xi_{F_{2}}}$ (the parameter, $\xi_{F_{2}}$ will be defined in a moment). This two component hidden sector dark matter model has been briefly discussed previously in ref.[15]. Our purpose here is to study it in more detail taking into account the tentative dark matter signal coming from the CRESST-II and CDMS/Si experiments[7] as well as the updated results from CoGeNT[6].

The differential scattering rate for $F_{j}$ on a target nuclei, $A$, is given by ${ }^{6}$ :

$$
\frac{d R}{d E_{R}}=N_{T} n_{F_{j}} \int_{|\mathbf{v}|>v_{\text {min }}}^{\infty} \frac{d \sigma}{d E_{R}} \frac{f_{F_{j}}\left(\mathbf{v}, \mathbf{v}_{E}\right)}{k}|\mathbf{v}| d^{3} v
$$

where the integration limit, $v_{m i n}$, is defined by the kinematic relation:

$$
v_{m i n}=\sqrt{\frac{\left(m_{A}+m_{F_{j}}\right)^{2} E_{R}}{2 m_{A} m_{F_{j}}^{2}}} .
$$

In Eq.(9), $N_{T}$ is the number of target nuclei per $\mathrm{kg}$ of detector and $n_{F_{j}}$ is the number density of the halo dark matter particles $F_{j}$ at the Earth's location. This number density can be expressed in terms of the halo mass fraction of $F_{j}, \xi_{F_{j}}$, and total mass density, $\rho_{d m}$ via $n_{F_{j}}=\rho_{d m} \xi_{F_{j}} / m_{F_{j}}$ (we set $\rho_{d m}=0.3 \mathrm{GeV} / \mathrm{cm}^{3}$ ). Also in Eq.(9) $\mathbf{v}$ denotes the velocity of the halo particles relative to the Earth and $\mathbf{v}_{E}$ the velocity of the Earth relative to the galactic halo ${ }^{7}$. The halo distribution function, in the reference frame of the Earth, is then given by the Maxwellian distribution:

$$
\frac{f_{F_{j}}\left(\mathbf{v}, \mathbf{v}_{E}\right)}{k}=\left(\pi v_{0}^{2}\left[F_{j}\right]\right)^{-3 / 2} \exp \left(\frac{-\left(\mathbf{v}+\mathbf{v}_{E}\right)^{2}}{v_{0}^{2}\left[F_{j}\right]}\right) \text {. }
$$

The integral, Eq.(9), can be simplified in terms of error functions[14, 26] and solved numerically. Detector resolution effects can be incorporated by convolving the rate with a Gaussian, as was done for the mirror dark matter case in[11].

\footnotetext{
${ }^{6}$ The upper limit of integration in Eq.(9) is taken as infinity since we are dealing with dark matter particles with potentially significant self-interactions. The self-interactions can prevent particles in the high velocity tail of the Maxwellian distribution from escaping the galaxy.

${ }^{7}$ In all numerical work we include an estimate of the Sun's peculiar velocity so that $\left\langle\left|\mathbf{v}_{E}\right|\right\rangle=$ $v_{\text {rot }}+12 \mathrm{~km} / \mathrm{s}$.
} 


\section{Direct detection of hidden sector dark matter}

As discussed earlier, the two component hidden sector model supposes dark matter arises from a hidden sector with two components $F_{1}$ and $F_{2}$. With $m_{F_{1}} \lesssim 5 \mathrm{GeV}$, the existing direct detection experiments depend on three parameters: $m_{F_{2}}, \epsilon \sqrt{\xi_{F_{2}}}, \bar{m}$. It was shown in ref.[15] that with $\bar{m}=1 \mathrm{GeV}$ and $v_{\text {rot }}=240 \mathrm{~km} / \mathrm{s}$ (fixed as a specific example) this model could provide a reasonable fit to the DAMA annual modulation signal and measured CoGeNT spectrum. Since that time, the measured CoGeNT spectrum has undergone a significant 'surface event correction'[6]. Additionally, CRESST-II and CDMS/Si have announced results suggesting a tentative dark matter signal. Our purpose now is to re-examine the two component hidden sector model in light of these new experimental developments. We shall also extend our previous analysis by studying a wider range of $\bar{m}, v_{\text {rot }}$ values. In particular we take $\bar{m} / \mathrm{GeV}=1.0$ and 3.0 and two values for the rotational velocity, $v_{\text {rot }}=200$ $\mathrm{km} / \mathrm{s}$ and $v_{\text {rot }}=240 \mathrm{~km} / \mathrm{s}$. Although $v_{\text {rot }}=240 \mathrm{~km} / \mathrm{s}$ is representative of recent measurements of the local rotational velocity of the Milky Way[31] there are significant uncertainties in this quantity and it is useful to see how things change when $v_{\text {rot }}$ is varied.

\section{The CRESST-II experiment}

The CRESST-II experiment has collected a $730 \mathrm{~kg}$-day exposure of a $\mathrm{CaWO}_{4}$ target[7]. The data arises from eight detector modules, with recoil energy thresholds (keV) of $10.2,12.1,12.3,12.9,15.0,15.5,16.2,19.0$. The binned CRESST-II data is given in table 1 . For the CRESST-II analysis, we define $\chi^{2}$ :

$$
\chi^{2}\left(m_{F_{2}}, \epsilon \sqrt{\xi_{F_{2}}}, \bar{m}\right)=\sum_{i=1}^{5}\left[\frac{R_{i}+B_{i}-d a t a_{i}}{\delta \operatorname{data}_{i}}\right]^{2}
$$

where $R_{i}$ is the predicted rate and $B_{i}$ is the estimated background in the $i^{\text {th }}$ energy bin. The relevant rates, $R_{i}$, for the CRESST-II experiment are computed from Eq.(9). The resolution is included using $\sigma_{\text {res }}=0.3 \mathrm{keV}[7]$. Also included are the detection efficiencies, $\epsilon_{f}$, for the three target elements: $\epsilon_{f}=0.9$ for $O, W$ and $\epsilon_{f}=1.0$ for $\mathrm{Ca}$, which take into account their acceptance region. No energy scale uncertainty is considered for CRESST-II.

\section{The DAMA experiment}

The DAMA/NaI and DAMA/LIBRA experiments have accumulated data from a large exposure [1.17 ton-year] of a NaI target[2, 3]. Analysis of this data has yielded an annual modulation with phase consistent with dark matter expectations at around $8.9 \sigma$ C.L. We analyse this annual modulation signal in the energy range $2-8 \mathrm{keVee}$ using 12 bins of width $0.5 \mathrm{keVee[3].} \mathrm{The} \mathrm{detector} \mathrm{resolution,} \mathrm{obtained} \mathrm{from} \mathrm{ref.[32],}$ has been included. The quenching factors $q_{N a}, q_{I}$, which set the nuclear recoil energy 


\begin{tabular}{ccc}
\hline \hline Bin / keV & Total events & Estimated background \\
\hline $10.2-13.0$ & 9 & 3.2 \\
$13-16$ & 15 & 6.1 \\
$16-19$ & 11 & 7.0 \\
$19-25$ & 12 & 11.5 \\
$25-40$ & 20 & 20.1 \\
\hline \hline
\end{tabular}

Table 1: CRESST-II data: total number of events and estimated background.

scale, are uncertain $\left[\mathrm{keVee}=\mathrm{keV}_{N R} / q\right]$. Following the mirror dark matter analysis of [11], we consider a range of possible values for the quenching factors: ${ }^{8}$

$$
q_{N a}=0.28 \pm 0.08, \quad q_{I}=0.12 \pm 0.08 .
$$

Assuming that $q_{I}, q_{N a}$ are energy independent, we minimize $\chi^{2}$ varying $q_{N a}, q_{I}$ over the above range. Values of $q_{I}, q_{N a}$ outside this range are possible and have been discussed in the literature. For example, the higher values $q_{N a} \approx 0.6, q_{I} \approx 0.3$ have been suggested by Tretyak[35]. Although the possibility of such high quenching factors are not specifically considered in our numerical work, we note here that higher values for the quenching factors generally move the DAMA allowed region to lower values of $\epsilon \sqrt{\xi_{F_{2}}}, m_{F_{2}}$.

\section{The CoGeNT experiment}

The CoGeNT collaboration has recently presented data corresponding to an exposure of $0.33 \times 807 \mathrm{~kg}$-days in a Germanium target[6]. This update of their earlier exposure[5] includes an important correction for surface events which have not been excluded by their rise time cut[6]. The efficiency corrected and surface event corrected CoGeNT data is obtained from figure 21 of ref.[6]. This data is analysed using 15 bins of width 0.1 keVee in the region $0.5-2.0$ keVee taking into account the advertised detector resolution. We allow for a constant background, which we fit to the data in this energy range. Uncertainties in energy scale are included by minimizing the $\chi^{2}$ for CoGeNT over the variation in quenching factor, $q_{G e}=0.21 \pm 0.04$.

\section{The CDMS/Si experiment}

The CDMS/Si experiment has observed three dark matter candidate events in an array of silicon detectors[8]. These three events have nominal recoil energies: 8.2 $\mathrm{keV}, 9.5 \mathrm{keV}$ and $12.3 \mathrm{keV}$. A $\chi^{2}$ analysis cannot be used given the low number of events. Instead, the likelihood function is constructed using the extended maximum

\footnotetext{
${ }^{8}$ In our analysis we neglect the possibility of channeling (scatterings with $q \simeq 1$ ). A theoretical study[33] and also recent experimental work[34] both suggest that the channeling fraction is probably small. Nevertheless we should keep in mind that even a channeling fraction as low as a few percent can significantly shift the DAMA favored regions of parameter space to lower $\epsilon \sqrt{\xi_{F_{2}}}$ values.
} 
likelihood formalism[36]. This has the form:

$$
\mathcal{L}(\mathbf{p})=\left[\Pi_{i} \frac{d n\left(E_{R}^{i}\right)}{d E_{R}}\right] \exp [-\mathcal{N}(\mathbf{p})]
$$

where the unknown parameters are denoted by the vector p. Here, $d n\left(E_{R}^{i}\right) / d E_{R}$ is the expected event rate evaluated at the recoil energy for the three observed events, $i=1, \ldots, 3$, . The total number of expected events is

$$
\mathcal{N}(\mathbf{p})=\int \frac{d n}{d E_{R}} d E_{R}
$$

where the integration limits cover the acceptance recoil energy region. The expected event rate, $d n / d E_{R}$, is the rate $d R / d E_{R}$, Eq.(9), convolved with a Gaussian to take into account the resolution ${ }^{9}$ and multiplied by the detection efficiency, $\epsilon_{f}\left(E_{R}\right)$ (obtained from ref.[8] for the $140.2 \mathrm{~kg}$-day exposure).

There are some indications[8] that the recoil energy calibration is around $10 \%$ higher than nominally used, with some uncertainty. We have thus scaled up the energies by a factor, $f=1.1$ and considered an energy calibration uncertainty of $\pm 10 \%$, i.e. $f=1.1 \pm 0.1$. The profile likelihood function, $\mathcal{L}_{P}$, is then obtained by maximizing $\mathcal{L}$ over this range of $f$ for each value of the parameters: $m_{F_{2}}, \epsilon \sqrt{\xi_{F_{2}}}$. The favored region for the parameters $m_{F_{2}}, \epsilon \sqrt{\xi_{F_{2}}}$ can then be obtained from the condition:

$$
\ln \mathcal{L}_{P} \geq \ln \mathcal{L}_{P \max }-\Delta \ln \mathcal{L}_{P}
$$

We set $2 \Delta \ln \mathcal{L}_{p}=5.99$ which corresponds to $95 \%$ C.L. for 2 parameters[37]. In this analysis we neglect the background contribution. This is justified given that the total background is estimated to be much less than 1 event for the CDMS exposure (in the energy region of interest, $E_{\text {threshold }} \leq E_{R} \leq 20 \mathrm{keV}$ )[8].

\section{The analysis}

We summarize the $\chi^{2}(\mathrm{~min})$ values for the relevant data sets from each experiment for the two chosen $\bar{m}$ values in table 2 for $v_{\text {rot }}=200 \mathrm{~km} / \mathrm{s}$ and table 3 for $v_{\text {rot }}=240$ $\mathrm{km} / \mathrm{s}$. The $95 \%$ C.L. favored region of parameter space is bounded by the contours where $\chi^{2}\left(m_{F_{2}}, \epsilon \sqrt{\xi_{F_{2}}}\right)=\chi_{\text {min }}^{2}+5.99$. In figure 1 we plot the favored region of parameter space for DAMA, CoGeNT, CRESST-II and CDMS $/$ Si for $v_{\text {rot }}=200 \mathrm{~km} / \mathrm{s}$ and with reference value of $\bar{m}: \bar{m}=1.0 \mathrm{GeV}$ (figure 1a), $\bar{m}=3.0 \mathrm{GeV}$ (figure $1 \mathrm{~b}$ ). In figure 2 we repeat the exercise, but with $v_{\text {rot }}=240 \mathrm{~km} / \mathrm{s}$.

\footnotetext{
${ }^{9}$ In the absence of resolution measurements, we take $\sigma_{r e s}=0.1 \mathrm{keV}$.
} 


\begin{tabular}{cccl}
\hline \hline Experiment & $\chi^{2}$ (min)/d.o.f. & $\bar{m} / \mathrm{GeV}$ & Best fit parameters \\
\hline DAMA (annual mod.) & $5.8 / 10$ & $\bar{m}=1.0$ & $m_{F_{2}}=56.0 \mathrm{GeV}, \epsilon \sqrt{\xi_{F_{2}}}=7.2 \times 10^{-9}$ \\
CoGeNT (spectrum) & $9.4 / 12$ & $\bar{m}=1.0$ & $m_{F_{2}}=36.0 \mathrm{GeV}, \epsilon \sqrt{\xi_{F_{2}}}=4.9 \times 10^{-9}$ \\
CRESST (spectrum) & $0.1 / 3$ & $\bar{m}=1.0$ & $m_{F_{2}}=80.0 \mathrm{GeV}, \epsilon \sqrt{\xi_{F_{2}}}=4.9 \times 10^{-9}$ \\
CDMS (spectrum) & & $\bar{m}=1.0$ & $m_{F_{2}}=59.0 \mathrm{GeV}, \epsilon \sqrt{\xi_{F_{2}}}=2.4 \times 10^{-9}$ \\
\hline DAMA (annual mod.) & $6.1 / 10$ & $\bar{m}=3.0$ & $m_{F_{2}}=58.0 \mathrm{GeV}, \epsilon \sqrt{\xi_{F_{2}}}=7.8 \times 10^{-9}$ \\
CoGeNT (spectrum) & $10.5 / 12$ & $\bar{m}=3.0$ & $m_{F_{2}}=44.0 \mathrm{GeV}, \epsilon \sqrt{\xi_{F_{2}}}=5.5 \times 10^{-9}$ \\
CRESST (spectrum) & $0.3 / 3$ & $\bar{m}=3.0$ & $m_{F_{2}}=74.0 \mathrm{GeV}, \epsilon \sqrt{\xi_{F_{2}}}=5.0 \times 10^{-9}$ \\
CDMS (spectrum) & & $\bar{m}=3.0$ & $m_{F_{2}}=49.0 \mathrm{GeV}, \epsilon \sqrt{\xi_{F_{2}}}=2.4 \times 10^{-9}$ \\
\hline \hline
\end{tabular}

Table 2: Summary of $\chi^{2}(\mathrm{~min})$ for the relevant data sets from the DAMA, CoGeNT, CRESST-II and CDMS/Si experiments for two reference $\bar{m}$ values and $v_{\text {rot }}=200$ $\mathrm{km} / \mathrm{s}$.

\begin{tabular}{cccc}
\hline \hline Experiment & $\chi^{2}$ min)/d.o.f. & $\bar{m} / \mathrm{GeV}$ & Best fit parameters \\
\hline DAMA (annual mod.) & $5.0 / 10$ & $\bar{m}=1.0$ & $m_{F_{2}}=42.0 \mathrm{GeV}, \epsilon \sqrt{\xi_{F_{2}}}=7.4 \times 10^{-9}$ \\
CoGeNT (spectrum) & $9.7 / 12$ & $\bar{m}=1.0$ & $m_{F_{2}}=28.5 \mathrm{GeV}, \epsilon \sqrt{\xi_{F_{2}}}=4.7 \times 10^{-9}$ \\
CRESST (spectrum) & $0.2 / 3$ & $\bar{m}=1.0$ & $m_{F_{2}}=57.7 \mathrm{GeV}, \epsilon \sqrt{\xi_{F_{2}}}=4.1 \times 10^{-9}$ \\
CDMS (spectrum) & & $\bar{m}=1.0$ & $m_{F_{2}}=35.0 \mathrm{GeV}, \epsilon \sqrt{\xi_{F_{2}}}=2.1 \times 10^{-9}$ \\
\hline DAMA (annual mod.) & $6.1 / 10$ & $\bar{m}=3.0$ & $m_{F_{2}}=46.0 \mathrm{GeV}, \epsilon \sqrt{\xi_{F_{2}}}=9.2 \times 10^{-9}$ \\
CoGeNT (spectrum) & $10.7 / 12$ & $\bar{m}=3.0$ & $m_{F_{2}}=36.5 \mathrm{GeV}, \epsilon \sqrt{\xi_{F_{2}}}=5.5 \times 10^{-9}$ \\
CRESST (spectrum) & $0.4 / 3$ & $\bar{m}=3.0$ & $m_{F_{2}}=39.8 \mathrm{GeV}, \epsilon \sqrt{\xi_{F_{2}}}=4.9 \times 10^{-9}$ \\
CDMS (spectrum) & & $\bar{m}=3.0$ & $m_{F_{2}}=27.0 \mathrm{GeV}, \epsilon \sqrt{\xi_{F_{2}}}=2.3 \times 10^{-9}$ \\
\hline \hline
\end{tabular}

Table 3: Summary of $\chi^{2}(\min )$ for the relevant data sets from the DAMA, CoGeNT, CRESST-II and CDMS/Si experiments for two reference $\bar{m}$ values and $v_{\text {rot }}=240$ $\mathrm{km} / \mathrm{s}$. 


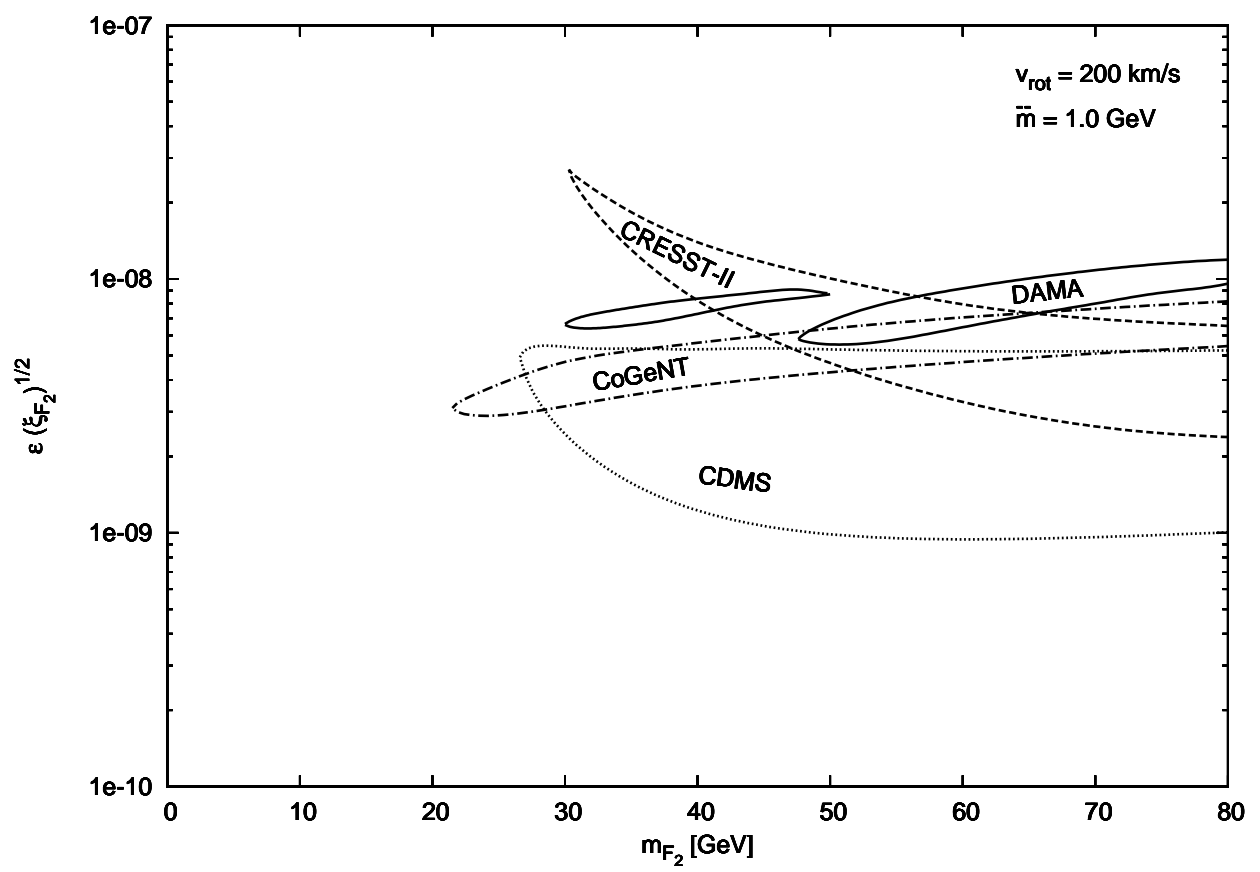

Figure 1a: DAMA (solid line), CoGeNT (dashed-dotted), CRESST-II (dashed) and CDMS/Si (dotted) favored regions of parameter space [all at 95\% C.L.] in the two component hidden sector model. The reference point $v_{\text {rot }}=200 \mathrm{~km} / \mathrm{s}$ and $\bar{m}=1.0 \mathrm{GeV}$ is assumed.

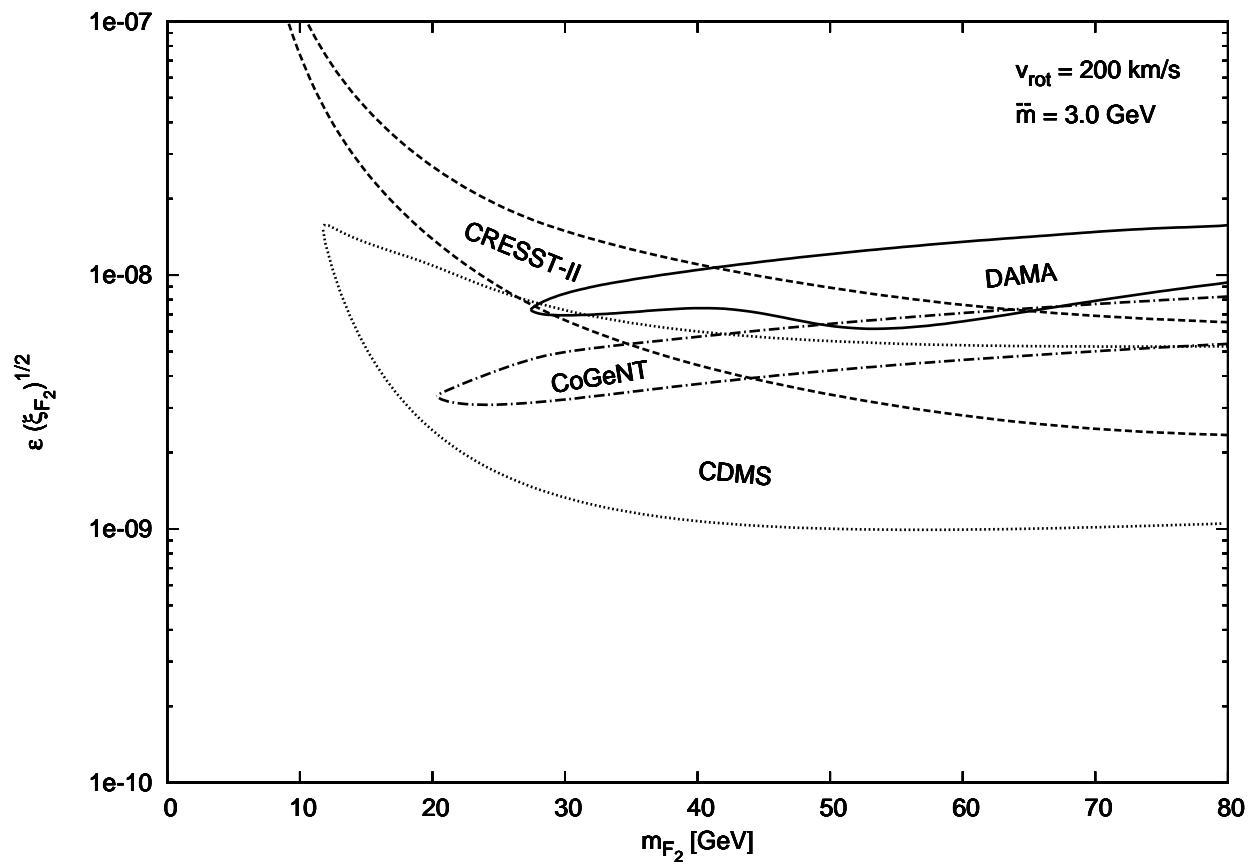

Figure 1b: Same as figure 1a, except that $\bar{m}=3.0 \mathrm{GeV}$ is assumed. 


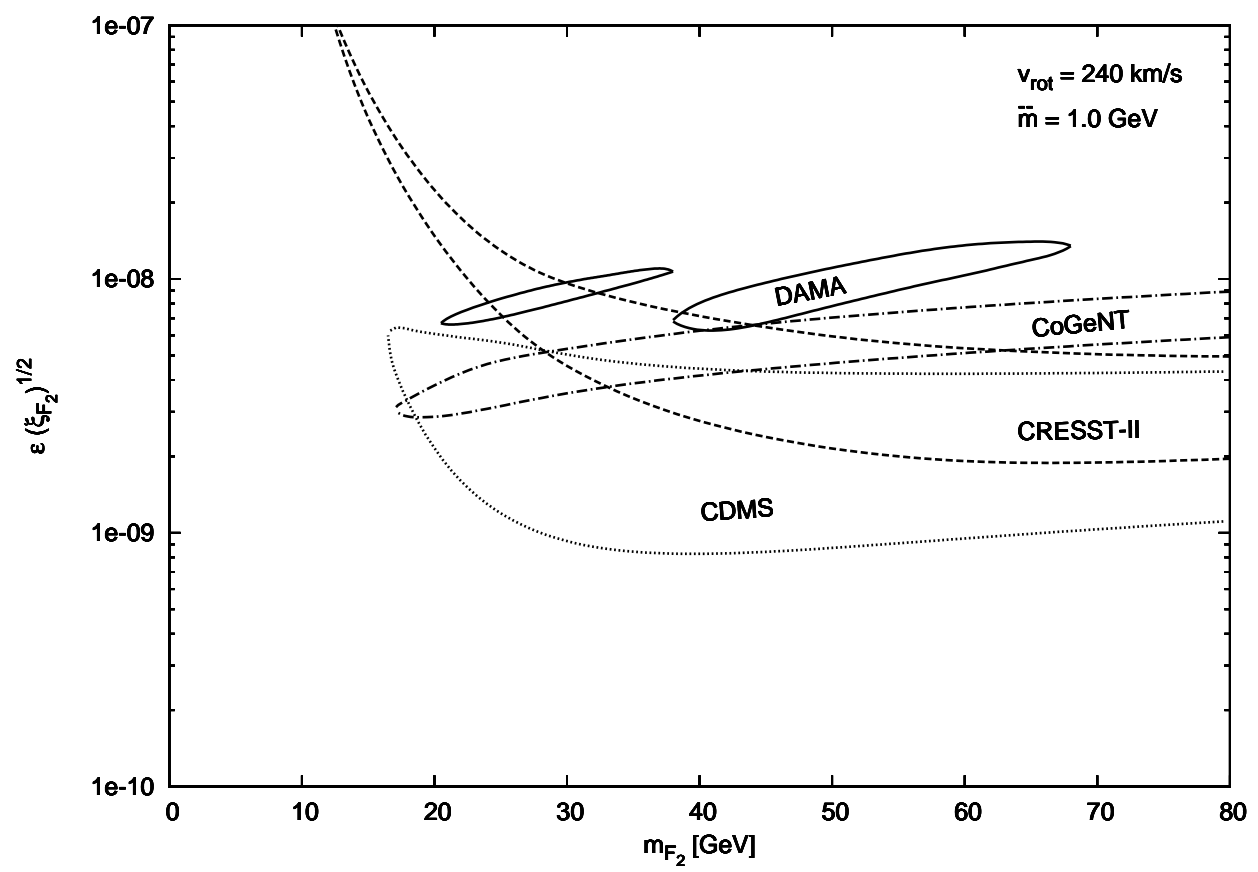

Figure 2a: DAMA (solid line), CoGeNT (dashed-dotted), CRESST-II (dashed) and CDMS/Si (dotted) favored regions of parameter space [all at 95\% C.L.] in the two component hidden sector model. The reference point $v_{\text {rot }}=240 \mathrm{~km} / \mathrm{s}$ and $\bar{m}=1.0 \mathrm{GeV}$ is assumed.

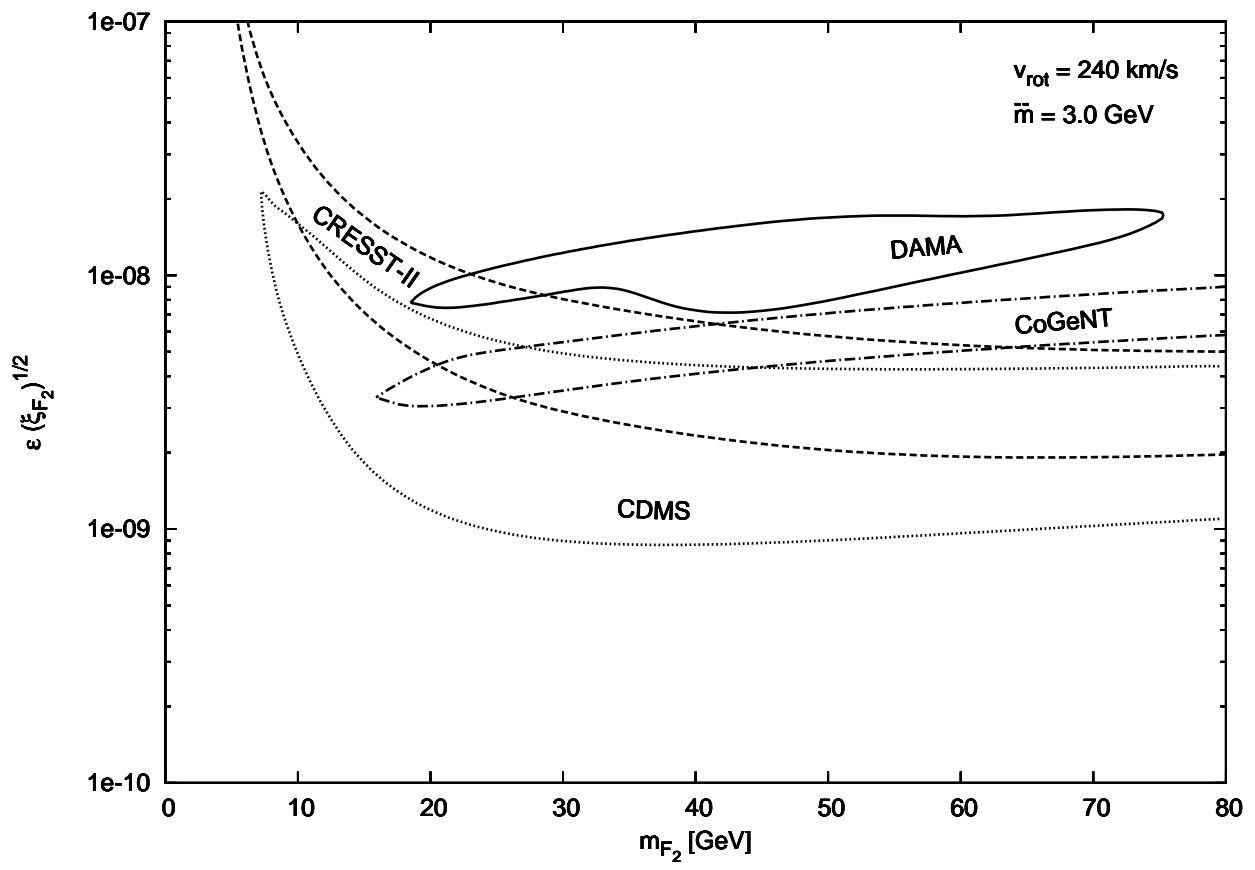

Figure 2b: Same as figure 2a, except that $\bar{m}=3.0 \mathrm{GeV}$ is assumed. 
The figures demonstrate that there are quite substantial regions of parameter space where each experiment can be explained within this hidden sector framework. For DAMA, the signal is dominated by $F_{2}-N a$ scattering if $m_{F_{2}} \lesssim 40 \mathrm{GeV}$, while if $m_{F_{2}} \gtrsim 40 \mathrm{GeV}$ then both $F_{2}-N a$ and $F_{2}-I$ scattering conspire to produce the signal. Also, the signal arises from scattering of target nuclei with $F_{2}$ particles from the body of their Maxwellian halo distribution (rather than, say, the tail) and thus is reasonably stable, and shows fairly mild dependence on the velocity dispersion i.e. on $\bar{m}$.

For CoGeNT, the spectrum is consistent with $d R / d E_{R} \propto 1 / E_{R}^{2}$, predicted from the energy dependence of the Rutherford cross-section. This is the reason why the CoGeNT spectrum is reproduced for a large range of $F_{2}$ mass. In other words, the shape of the CoGeNT spectrum arises from dynamics rather than kinematic effects in this model. Again there is little dependence on the $\bar{m}$ parameter.

In the case of CRESST-II, the dominant signal contribution arises from $\mathrm{F}_{2}-\mathrm{Ca}$ scattering for $m_{F_{2}} \sim 50 \mathrm{GeV}$. For such masses, $F_{2}$ particles in the body of their Maxwellian halo distribution can scatter to produce nuclear recoils above the $10 \mathrm{keV}$ threshold. As the mass of $F_{2}$ is lowered, $m_{F_{2}} \lesssim 40-50 \mathrm{GeV}$, a recoil above threshold can only occur for $F_{2}$ particles in the tail of the Maxwellian halo distribution. For this reason, there is quite a bit of dependence of the CRESST-II allowed region on the velocity dispersion, i.e. $\bar{m}$ in this mass range.

The figures show that the allowed region of parameters favored by DAMA, CoGeNT, CRESST-II and CDMS/Si are very similar with a significant degree of overlap. This occurs ignoring a variety of possible systematic effects, such as the possibility of channeled events in DAMA (which can lower the DAMA favored values of $\epsilon \sqrt{\xi_{F_{2}}}$ ), and uncertainties in the surface event correction factor for CoGeNT (which could raise or lower the CoGeNT favored values of $\epsilon \sqrt{\xi_{F_{2}}}$ ). Thus realistically there is quite a large region of parameter space that is possible, much larger than the overlapping allowed region, even assuming all four experiments have detected dark matter interactions. It is difficult, though, to quantify all the possible systematic effects and do an exhaustive analysis. Instead, we shall hope to gain some insight by examining a particular point in parameter space.

\section{An example near the combined best fit of DAMA, CoGeNT, CRESST-II and CDMS/Si}

In view of potential systematic uncertainties between the experiments we shall resist the temptation to fit the combined DAMA, CoGeNT, CRESST-II and CDMS/Si data. Instead we shall consider an example reference point $P 1$ located near the overlapping allowed regions indicated in figure 1a.

$$
P 1: m_{F_{2}}=50 \mathrm{GeV}, \epsilon \sqrt{\xi_{F_{2}}}=5.7 \times 10^{-9}, \bar{m}=1.0 \mathrm{GeV}, v_{\text {rot }}=200 \mathrm{~km} / \mathrm{s} .
$$

The DAMA annual modulation signal for this example is given in figure $3 \mathrm{a}$, the CoGeNT spectrum in figure 3b, and CRESST-II spectrum in figure 3c. 


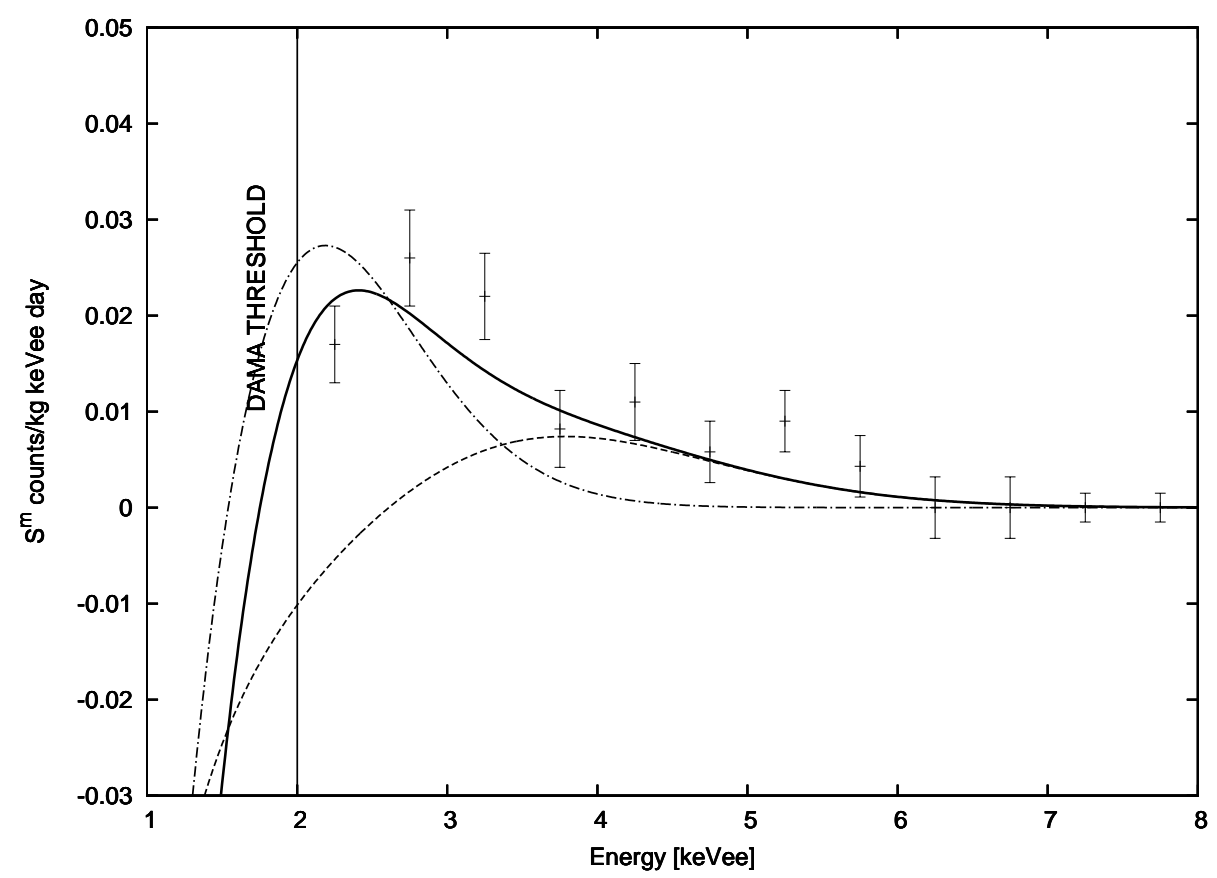

Figure 3a: DAMA annual modulation spectrum for two component hidden sector dark matter with parameters $P 1$ (solid line). The separate contributions from scattering off sodium (dotted line) and Iodine (dashed-dotted line) are also shown. In this example $q_{N a}=0.36, q_{I}=0.20$.

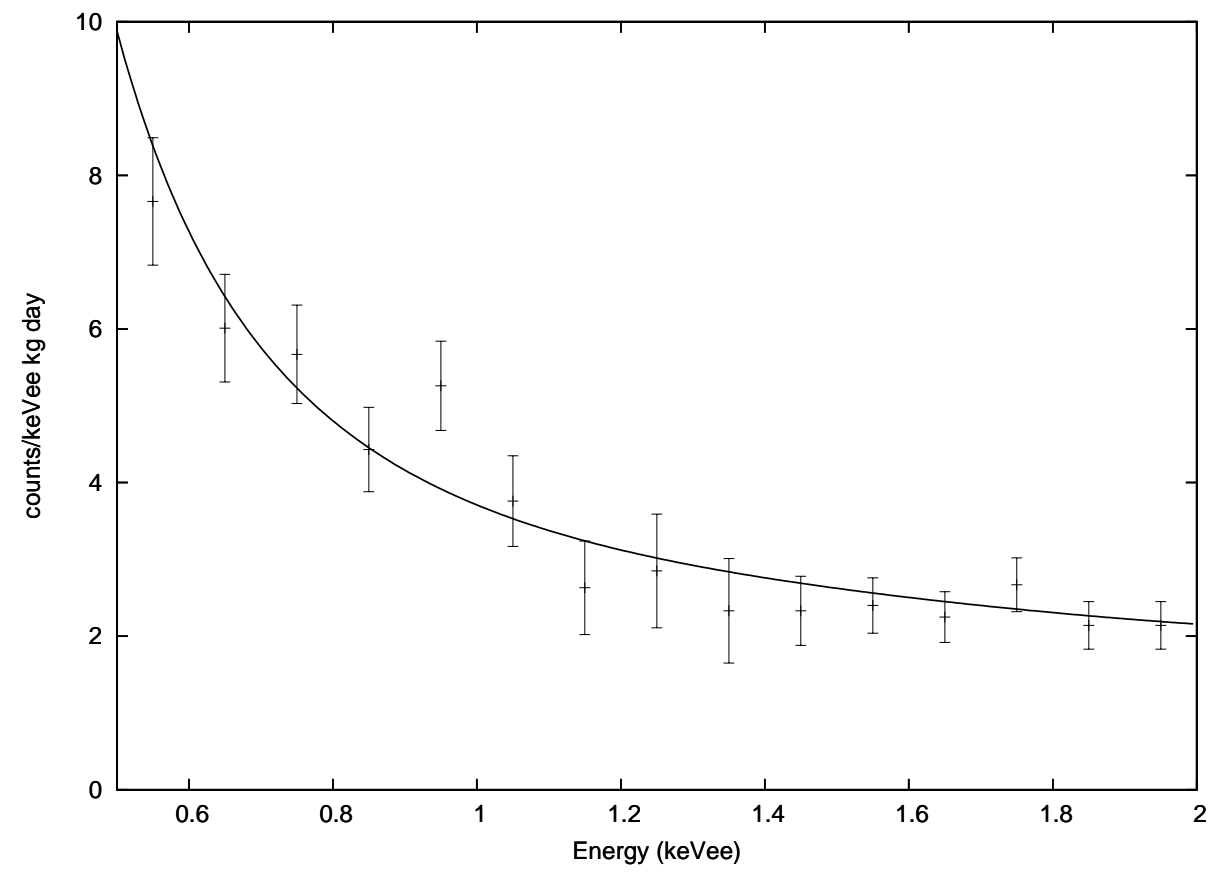

Figure 3b: CoGeNT spectrum for two component hidden sector dark matter with parameters $P 1$. In this example $q_{G e}=0.17$. 


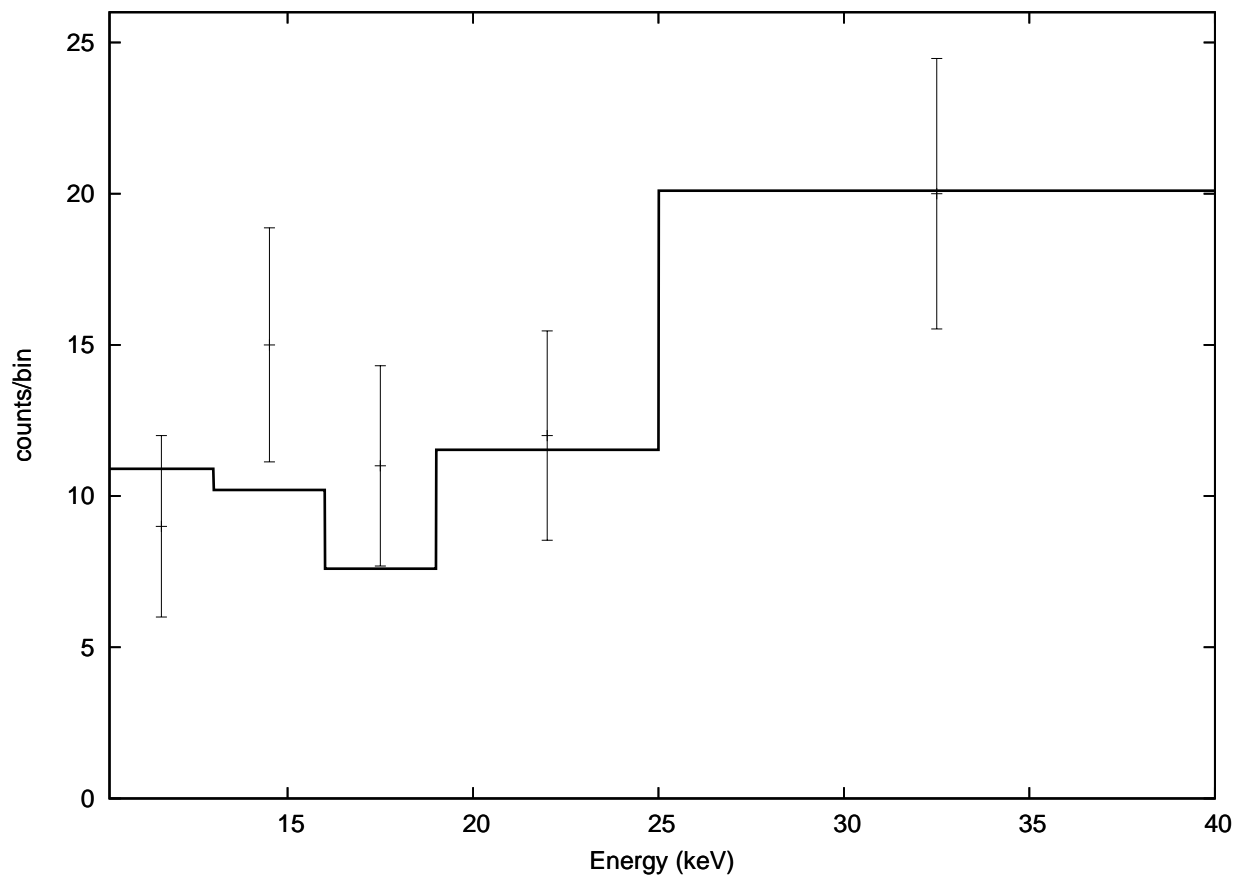

Figure 3c: CRESST spectrum for two component hidden sector dark matter for the example $P 1$.

Figures 3 clearly demonstrates that two component hidden sector dark matter can simultaneously explain DAMA, CoGeNT and CRESST-II data. The apparent bump in the DAMA data can be reproduced extremely well and the minimal model predicts a change in sign of the DAMA annual modulation signal at low energies. Note though, that if there are additional particles, $F_{3}$, of intermediate mass $\sim 20$ $\mathrm{GeV}$, then their positive contribution to the annual modulation can outweigh the negative contribution from $F_{2}$. An example, given in the context of mirror dark matter with $F_{2} \sim F e^{\prime}$ and $F_{3} \sim O^{\prime}$, was discussed in ref.[11]. Figure 3b indicates that the shape of the CoGeNT spectrum is consistent with the $d R / d E_{R} \propto 1 / E_{R}^{2}$ energy dependence of the Rutherford cross-section. The event rate is predicted to continue to rise as $\sim 1 / E_{R}^{2}$ as the recoil energy is reduced below the current CoGeNT threshold, until the threshold of a lighter component is reached, whereby the rate can jump even higher. These effects can be probed by TEXONO, C-4, CDMSlite and possibly other experiments.

Future data from DAMA, CoGeNT, CRESST-II and other experiments will obviously be able to constrain the parameter space within this hidden sector framework. As discussed recently[38], a particularly striking diurnal modulation signal should be observable for a detector located in the southern hemisphere, and perhaps even in the northern hemisphere at low latitudes, such as detectors in Jin-Ping underground laboratory. In the meantime, we must rely on annual modulation and spectrum data. 
In figure 4 we give the predicted spectrum for DAMA/LIBRA for the reference point, $P 1$. Figure 4 also shows the 'single hit' event rate recorded in DAMA/LIBRA[3]. The figure indicates that the sharp rise in the predicted dark matter interaction rate could potentially be differentiated from the background if the DAMA threshold is lowered below 2 keVee. In figure 5 we show the predicted annual modulation spectrum for CoGeNT for the same reference point, $P 1$. Clearly, the initial annual modulation amplitude measured by CoGeNT to be $A \approx 0.46 \pm 0.17 \mathrm{cpd} / \mathrm{kg} / \mathrm{keVee}$ averaged over the energy range: $0.5<E$ (keVee) $<3.0$, is much larger than that predicted by our example point. Also, we find that the annual modulation changes sign at low energies. This feature is not supported by CoGeNT's initial measurement. However the energy where the modulation changes sign can be reduced if the mass of $F_{2}$ is lowered, which can alleviate this discrepancy. Alternatively, there can be an additional dark component, $F_{3}$, of mass $\sim 20 \mathrm{GeV}$. As discussed earlier in the context of DAMA, such a component can give a positive contribution to the annual modulation which can outweigh the negative contribution from $F_{2}$. Clearly future measurements of the annual modulation by CoGeNT, C-4, CDEX, and other experiments will be very important.

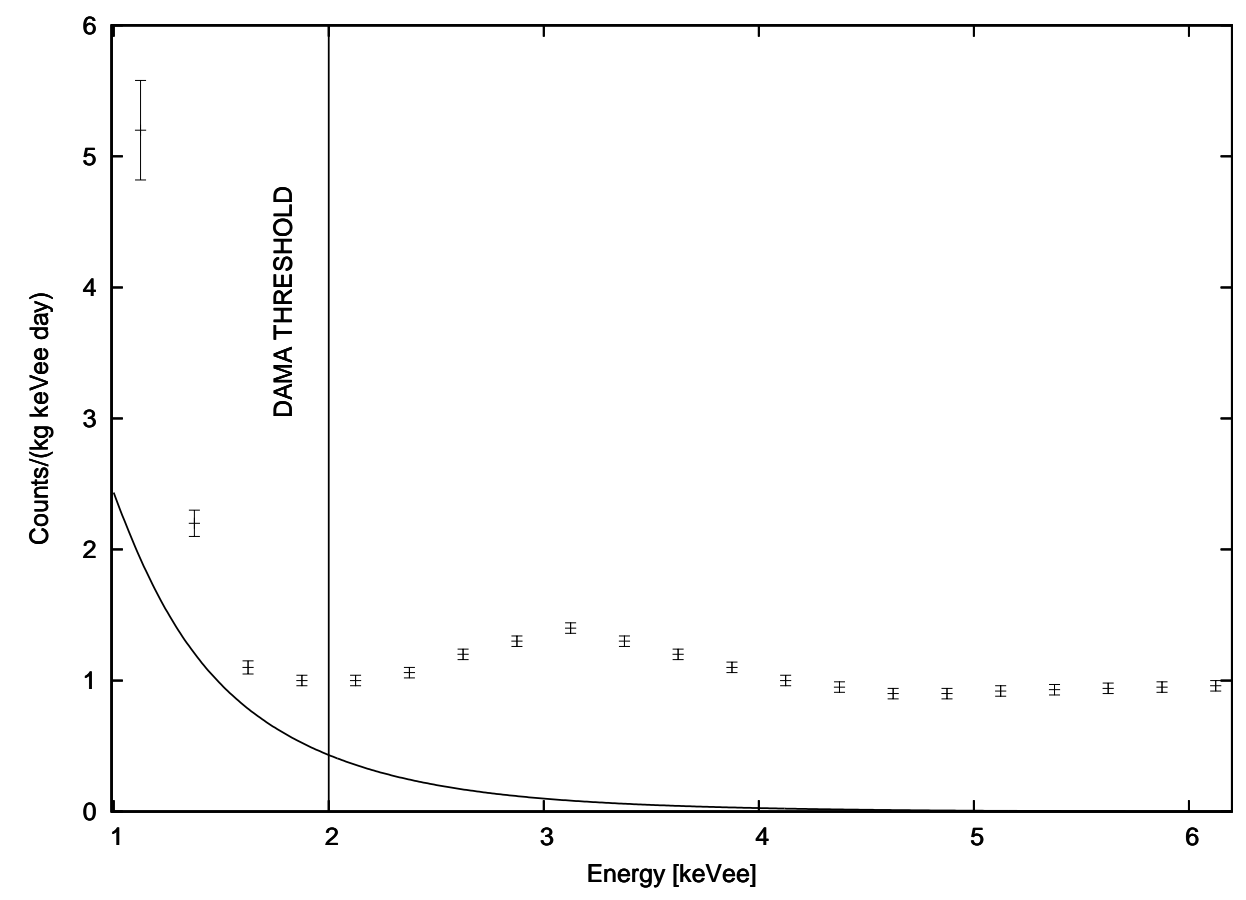

Figure 4: DAMA spectrum for hidden sector dark matter with parameters $P 1$. In this example $q_{N a}=0.36, q_{I}=0.20$. 


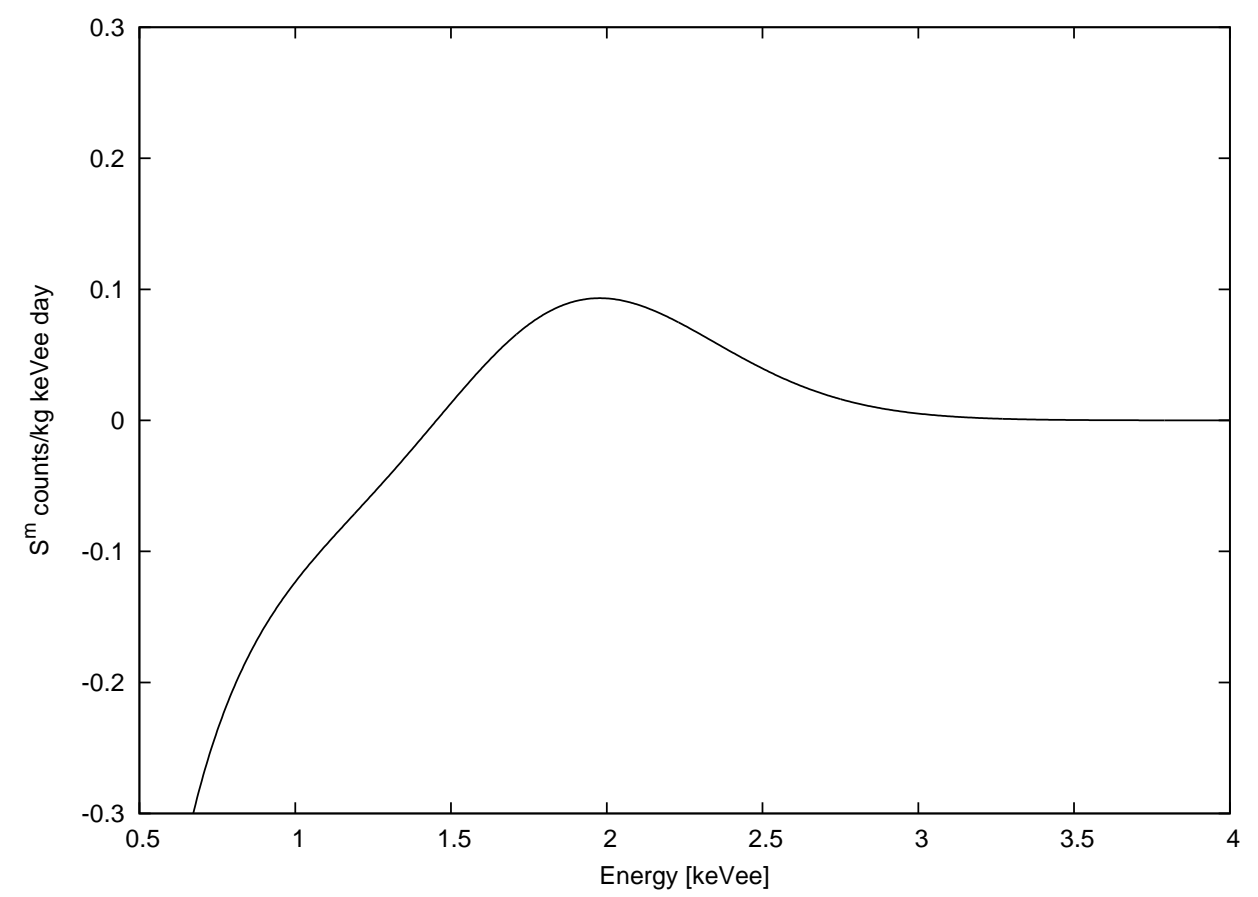

Figure 5: CoGeNT annual modulation spectrum for hidden sector dark matter with parameters $P 1$. In this example $q_{G e}=0.17$.

\section{XENON100 constraints}

The XENON100[29] experiment has reported null results in their dark matter search. Here, we examine the compatibility of the considered hidden sector dark matter model with these null results ${ }^{10}$. The constraints from the XENON100 experiments depend sensitively on the recoil energy threshold, which has significant uncertainty. The precise magnitude of this uncertainty is unknown, but the subject of active discussions[42, 43]. Given this situation, we examine the compatibility issue by estimating the energy threshold for which the parameter point $P 1$ can be excluded at 95\% C.L. Taking into account the relevant detection efficiencies, exposure time and detector resolution, we find that the energy threshold of the XENON100 experiment needs to be around $15 \mathrm{keV}$ for the point $P 1$ to be consistent at $95 \%$ C.L. This can be compared with the nominal threshold energy of $6.4 \mathrm{keV}$. Our example point assumed $\bar{m}=1.0 \mathrm{GeV}$. Raising $\bar{m}$ will increase the tension of hidden sector dark matter with the null results the XENON100 experiment. Lowering $\bar{m}$ can improve the situation,

\footnotetext{
${ }^{10}$ There are also lower threshold analysis by the XENON10[39] and CDMS collaborations[8]. However it has been argued[40] that neither analysis can exclude light dark matter (and by extension, hidden sector dark matter examined here, which has similar event rates at low energies) when systematic uncertainties are properly taken into account. Interestingly, a recent analysis[41] has found that the low energy CDMS data are actually fully consistent with CoGeNT's observed low energy excess rate, adding weight to the dark matter interpretation of this excess.
} 
but only moderately so. Although $P 1$ was just an example point, it seems that some level of tension exists between the null results of XENON100 and the hidden sector dark matter explanation of the DAMA, CoGeNT, CRESST-II and CDMS/Si experiments.

One can envisage several possible ways in which this tension might be alleviated. For example, it is possible that there is an issue with the calibration of the XENON100 apparatus. Determining the recoil energy scale in the XENON100 detector is nontrivial and it seems possible that this scale might be have a factor $\sim 2$ uncertainty[42, 43]. Another possibility is that $F_{2}$ has a somewhat lower mass than given in our example. This option would be especially relevant if we were to ignore the CRESST-II excess ${ }^{11}$. For example the parameter point:

$$
P 2: m_{F_{2}}=40 \mathrm{GeV}, \epsilon \sqrt{\xi_{F_{2}}}=5.7 \times 10^{-9}, \bar{m}=1.0 \mathrm{GeV}, v_{\text {rot }}=190 \mathrm{~km} / \mathrm{s}
$$

yields a $\chi^{2}($ dama $)=18.9$ for 12 data points and $\chi^{2}$ (cogent $)=22.8$ for 15 data points, but gives only around 1 event for the CRESST-II exposure. This is a reasonable fit for DAMA and CoGeNT, considering that the shape of the distributions are fit well, only the overall normalization is not [CoGeNT (DAMA) prefers slightly smaller (larger) $\epsilon \sqrt{\xi_{F_{2}}}$, with $\epsilon \sqrt{\xi_{F_{2}}}=5.7 \times 10^{-9}$ a compromise]. The different normalizations might easily be due to systematic effects not included in our analysis. Taking into account the relevant detection efficiencies, exposure time and detector resolution, we find that the energy threshold of the XENON100 experiment needs to be around $11.5 \mathrm{keV}$ for the point $P 2$ to be consistent at $95 \%$ C.L. ${ }^{12}$.

\section{Conclusion}

We have examined the data from the DAMA, CoGeNT, CRESST-II and CDMS/Si experiments in the context of multi-component hidden sector dark matter. The models considered feature a hidden sector with two or more stable particles charged under an unbroken $U(1)^{\prime}$ gauge interaction. The new gauge field can interact with the standard $U(1)_{Y}$ via renormalizable kinetic mixing, leading to Rutherford-type elastic scattering of the dark matter particles off ordinary nuclei. We examined the simplest generic model of this type, with a hidden sector composed of two stable particles, $F_{1}$ and $F_{2}$.

We have found that the two component hidden sector dark matter model can simultaneously explain the DAMA, CoGeNT, CRESST-II and CDMS/Si data. This explanation has some tension with the XENON100 experiment. The favored parameter regions are typically consistent with the most recent XENON100 results only if the XENON100 energy threshold is around a factor of two higher than given by the XENON100 collaboration.

\footnotetext{
${ }^{11}$ See [44] for a discussion of a subtle background effect which might potentially explain the CRESST-II low energy excess.

${ }^{12}$ We have checked that the example points $P 1$ and $P 2$ are consistent with the CDMS/Ge[30] data (taking a systematic uncertainty in energy scale of 20\%) and also the KIMS experiment[45].
} 


\section{Acknowledgments}

This work was supported by the Australian Research Council.

\section{References}

[1] S. Dodelson, "Modern cosmology," Amsterdam, Netherlands: Academic Pr. (2003) 440 p; G. Bertone, D. Hooper and J. Silk, Phys. Rept. 405, 279 (2005) [hep-ph/0404175].

[2] R. Bernabei et al. [DAMA Collaboration], Riv. Nuovo Cimento. 26, 1 (2003) [astro-ph/0307403]; Int. J. Mod. Phys. E13, 2127 (2004); Phys. Lett. B480, 23 (2000).

[3] R. Bernabei et al. [DAMA Collaboration], Eur. Phys. J. C56, 333 (2008) [arXiv:0804.2741]; Eur. Phys. J. C67, 39 (2010) [arXiv: 1002.1028].

[4] A. K. Drukier, K. Freese and D. N. Spergel, Phys. Rev. D33, 3495 (1986); K. Freese, J. A. Frieman and A. Gould, Phys. Rev. D37, 3388 (1988).

[5] C. E. Aalseth et al. [CoGeNT Collaboration], Phys. Rev. Lett. 106, 131301 (2011) [arXiv:1002.4703]; Phys. Rev. Lett. 107, 141301 (2011) [arXiv:1106.0650].

[6] C. E. Aalseth et al. [CoGeNT Collaboration], arXiv:1208.5737.

[7] G. Angloher et al., Eur. Phys. J. C 72, 1971 (2012) [arXiv:1109.0702].

[8] R. Agnese et al. [CDMS Collaboration], [arXiv:1304.4279].

[9] R. Foot, H. Lew and R. R. Volkas, Phys. Lett. B 272, 67 (1991); Mod. Phys. Lett. A 7, 2567 (1992).

[10] R. Foot, Int. J. Mod. Phys. D13, 2161 (2004) [astro-ph/0407623]; Int. J. Mod. Phys. A19 3807 (2004) [astro-ph/0309330]; P. Ciarcelluti, Int. J. Mod. Phys. D19, 2151 (2010) [arXiv: 1102.5530].

[11] R. Foot, Phys. Rev. D 86, 023524 (2012) [arXiv:1203.2387]; arXiv:1305.4316.

[12] R. Foot, Phys. Rev. D69, 036001 (2004) [hep-ph/0308254].

[13] R. Foot, Mod. Phys. Lett. A19, 1841 (2004) [astro-ph/0405362]; astroph/0403043; Phys. Rev. D74, 023514 (2006) [astro-ph/0510705]; Phys. Rev. D82, 095001 (2010) [arXiv: 1008.0685]; Phys. Lett. B692, 65 (2010) [arXiv: 1004.1424].

[14] R. Foot, Phys. Rev. D78, 043529 (2008) [arXiv: 0804.4518].

[15] R. Foot, Phys. Lett. B 703, 7 (2011) [arXiv:1106.2688]. 
[16] J. -W. Cui, H. -J. He, L. -C. Lu and F. -R. Yin, Phys. Rev. D 85, 096003 (2012) [arXiv:1110.6893].

[17] J. L. Feng, H. Tu and H. -B. Yu, JCAP 0810, 043 (2008) [arXiv:0808.2318]; J. L. Feng, M. Kaplinghat, H. Tu and H. -B. Yu, JCAP 0907, 004 (2009) [arXiv:0905.3039]; S. Andreas, M. D. Goodsell and A. Ringwald, Phys. Rev. D 87, 025007 (2013) [arXiv:1109.2869]; X. Chu, T. Hambye and M. H. G. Tytgat, JCAP 1205, 034 (2012) [arXiv:1112.0493].

[18] N. F. Bell, K. Petraki, I. M. Shoemaker and R. R. Volkas, Phys. Rev. D 84, 123505 (2011) [arXiv:1105.3730]; K. Petraki, M. Trodden and R. R. Volkas, JCAP 1202, 044 (2012) [arXiv:1111.4786]; K. Petraki and R. R. Volkas, arXiv:1305.4939 and references there-in.

[19] R. Foot, arXiv:1304.4717.

[20] Z. K. Silagadze, ICFAI U. J. Phys. 2, 143 (2009) [arXiv:0808.2595].

[21] R. Foot, Phys. Lett. B 699, 230 (2011) [arXiv:1011.5078].

[22] N. Fornengo, P. Panci and M. Regis, Phys. Rev. D 84, 115002 (2011) [arXiv:1108.4661].

[23] R. Foot and X-G. He, Phys. Lett. B267, 509 (1991).

[24] B. Holdom, Phys. Lett. B 166, 196 (1986).

[25] R. H, Helm, Phys. Rev. 104, 1466 (1956).

[26] J. D. Lewin and P. F. Smith, Astropart. Phys. 6, 87 (1996).

[27] R. Foot and R. R. Volkas, Phys. Rev. D70, 123508 (2004) [astro-ph/0407522].

[28] J. M. Cline, Z. Liu and W. Xue, Phys. Rev. D 85, 101302 (2012) [arXiv:1201.4858].

[29] E. Aprile et al. [XENON100 Collaboration], arXiv:1207.5988.

[30] Z. Ahmed et al. [CDMS-II Collaboration], Science 327, 1619 (2010) [arXiv:0912.3592].

[31] A. Brunthaler et al., arXiv:1102.5350.

[32] R. Bernabei et al. [DAMA Collaboration], Nucl. Instrum. Meth. A 592, 297 (2008) [arXiv:0804.2738].

[33] N. Bozorgnia, G.B. Gelmini and P. Gondolo, JCAP 1011, 019 (2010) [arXiv: 1006.3110]; JCAP 1011, 028 (2010) [arXiv: 1008.3676].

[34] J. I. Collar, arXiv:1302.0796. 
[35] V. I. Tretyak, Astropart. Phys. 33, 40 (2010) [arXiv:0911.3041].

[36] R. Barlow, Nucl. Instrum. Methods A 297, 496 (1990).

[37] J. Beringer et al. [Particle Data Group Collaboration], Phys. Rev. D 86, 010001 (2012).

[38] R. Foot, JCAP 1204, 014 (2012) [arXiv:1110.2908].

[39] J. Angle et al. (XENON10 Collaboration), arXiv: 1104.3088.

[40] J. I. Collar, arXiv:1103.3481; arXiv:1106.0653.

[41] J. I. Collar and N. E. Fields, arXiv:1204.3559.

[42] J. I. Collar, arXiv:1010.5187.

[43] R. Cerulli et al., arXiv:1201.4582.

[44] M. Kuzniak, M. G. Boulay and T. Pollmann, Astropart. Phys. 36, 77 (2012) [arXiv:1203.1576].

[45] S. K. Kim, on behalf of the KIMS collaboration, TAUP 2011 workshop, Munich, Germany Sep 5-9, 2011. 